\section{Kidney \\ Blood Pressure Research}

\title{
Impact of Self-Report and eGFR-Based Chronic Kidney Disease on the Risk of Chronic Kidney Disease-Related Complications and Geriatric Syndromes in Community-Dwelling Older Adults
}

\author{
Chia-Ter Chao a,b,c Yi-Hsuan Lee ${ }^{a, c} \quad$ Kuen-Cheh Yang ${ }^{a, c} \quad$ Jen-Kuei Peng ${ }^{a, c}$ \\ Chia-Ming Li ${ }^{a, c}$ Shih-I Chen ${ }^{a}$ Der-Sheng Han ${ }^{a, c}$ Jenq-Wen Huang ${ }^{b}$ \\ COGENT study group ${ }^{d}$
}

\begin{abstract}
aDepartment of Medicine, National Taiwan University Hospital BeiHu branch, Taipei, ${ }^{\text {bNephrology }}$ division, Department of Internal Medicine, National Taiwan University Hospital, Taipei, 'Geriatric and Community Medicine Research Center, National Taiwan University Hospital BeiHu branch, Taipei, ${ }^{d}$ COhort of GEriatric Nephrology in National Taiwan University Hospital, Taipei, Taiwan
\end{abstract}

\section{Key Words}

Chronic kidney disease $\bullet$ Estimated glomerular filtrate rate $\bullet$ Geriatrics $•$ Geriatric syndrome $•$ Hypoalbuminemia Inflammation $•$ Malnutrition

\begin{abstract}
Background/Aims: Awareness of chronic kidney disease (CKD) has been low among affected patients, particularly the older ones. However, whether such awareness is synonymous with the presence of laboratory-diagnosed CKD among older adults is currently unclear. Methods: We enrolled community-dwelling old adults ( $\geq 65$ years) who received health examinations between 2013 and 2016 from a regional metropolitan hospital. Clinical information and geriatric syndromes including depression, cognitive impairment, fall, quality of life, and visual disturbance were evaluated during the medical interview. We compared the differences in clinical features between those with and without self-reported or estimated glomerular filtration rate (eGFR)-based CKD and investigated their influences and interactions on the risk of CKD complications and geriatric syndromes. Results: Among the 2932 enrolled older adults (mean $73.4 \pm 7$ years), 93 (3\%) reported that they had CKD by history, while 306 (10\%) had an eGFR $<60 \mathrm{~mL} / \mathrm{min} / 1.73 \mathrm{~m}^{2}$ persisted for over 3 months. The prevalence of hyperlipidemia, body mass index, waist circumference, leukocyte count, and the incidence of fall differed only between those with and without eGFR-based CKD, but not between those with and
\end{abstract}




\section{Kidney Blood Pressure Research}

without self-reported CKD. A synergistic effect was found between self-reported and eGFRbased CKD regarding the CKD complication severity, including malnutrition (albumin), anemia (hemoglobin), dyslipidemia (serum cholesterol), and geriatric syndromes (cognitive and quality of life impairment). Multivariate regression analyses showed that self-reported CKD exhibited better predictive efficacy for lower serum albumin and hemoglobin than eGFR-based CKD, while the latter outperformed the former for predicting lower serum cholesterol and a higher risk of cognitive impairment. Conclusion: Among older adults, self-reported CKD may not be a surrogate for laboratory-diagnosed CKD and has an independent effect on CKD-related complications.

\section{Introduction}

Chronic kidney disease (CKD) is an important public health issue that affects $8 \%$ to $16 \%$ of the population worldwide and accounts for $82 \%$ excess increase in loss of life [1,2]. Despite its clinical significance, CKD awareness remains disproportionally low. Based on the National Health and Nutrition Examination Survey results, the awareness of CKD was surprisingly low among respondents with stage $1(3.5 \%)$ to $3(7.8 \%)$ CKD and increased significantly among those with stage 4 CKD [3]. Survey data from the Kidney Early Evaluation Program (KEEP) with more than 70,000 participants also disclosed that the awareness rate was 8.1\%, although one-fourth had laboratory-confirmed CKD [4]. Those with better socioeconomic status, more advanced CKD, more comorbidities (particularly DM and hypertension), or proteinuria were more likely to be aware of CKD compared to the others $[3,5]$. Inadequate CKD awareness predisposes these patients to late nephrologist referral, delayed institution of treatment directed against CKD related complications, and poor preparation for dialysis when they reach end stage kidney failure, all of which contribute to increased morbidity and mortality.

A core presumption behind the above arguments is that patients' self-report CKD agrees with their actual renal function status. However, the accuracy of self-reported comorbidities can vary between types of morbidity, educational status or even other socioeconomic factors. The issue of accuracy is particularly problematic for older adults, since their health literacy and the ability to choose appropriate terms for effective medical communication might be declining. Previous studies suggested that among older adults, the accuracy of certain patient-reported comorbid diseases was excellent, while others were poor. Bush et al. identified that self-report diabetes mellitus (DM), myocardial infarction, and stroke exhibited a high agreement ( $>90 \%$ ) with medically diagnosed ones in a cohort of communitydwelling elderly [6]. Another study showed that DM and hypertension could be accurately recognized through self-report questions, while chronic lung disease or other symptombased conditions could not [7]. The issue of inaccuracy in comorbidity reporting may also be prominent among patients from Asian countries; a prior study suggested that self-report hypertension was inaccurate in predicting the presence of hypertension in a cohort of Asian elderly [8].

Similar situation may be applicable to the recognition of CKD. When an older individual state that they have "CKD," what they mean can range from "I have remote kidney damage" (with uncertain current status) or "I have kidney lesions" (without references to renal function), to "I currently have abnormal kidney function or renal function decline," which is compatible with the official definition of CKD. Importantly, none of the above studies address the accuracy of self-report CKD among elderly. This leaves a research gap that should be filled urgently. It is also expected that CKD based on different identification criteria influence their ability to affect outcomes.

It remains unclear whether self-report CKD has different outcome influences compared to laboratory-confirmed CKD. Several studies suggested that chronic conditions and geriatric syndromes interacted synergistically with respect to their association with health outcomes 


\section{Kidney Blood Pressure Research}

Chao et al:: Self-Report and eGFR-Based CKD

among older adults [9], but others revealed that morbidity might be less informative for outcome prediction than geriatric syndromes [10]. The controversy in results from the existing literature may be related to the accuracy of the comorbidity of interest. We hypothesized that self-report and laboratory-based CKD might exhibit differential influences on the risk of having several types of geriatric syndromes among elderly. Using data from a large cohort of community-dwelling older adults, we investigated the clinical features that differ between those with and without self-reported or estimated glomerular filtration rate (eGFR)-based CKD, and the impact of these two assessment methods on patient outcomes, especially CKD-related complications.

\section{Materials and Methods}

\section{Enrolment of participants and data retrieval}

We prospectively identified all community-dwelling elderly patients ( $\geq 65$ years) who received health examinations between 2013 and 2016 from a regional hospital. Those who received multiple examinations during the study period would only have their first examination results analyzed.

After they received a health examination, we recorded their demographic data including age, gender, anthropometric parameters (body height, body weight, body mass index [BMI], waist circumference), history of substance use (smoking, alcohol consumption, and betel nuts), self-reported comorbidities, and family history of chronic illnesses. Self-reported comorbidities were verified with repeated query during the interview. Prior studies showed that the definition of self-report comorbidity was mostly based on faceto-face interview conducted by trained interviewers using standardized questions in domestic languages without descriptions about the nature of the cited conditions [6-8]. In the current study, we adopted the standardized methodology used by past reports assessing the accuracy of self-report morbidities.

Participants then underwent physical examinations for blood pressure (BP) and pulse rate (PR), followed by blood tests in the morning for hemogram, serum biochemistry (urea nitrogen [UN], creatinine, aspartate/alanine transaminase, cholesterol, glucose, and uric acid). Participants with abnormal laboratory findings, including an eGFR lower than $60 \mathrm{~mL} / \mathrm{min} / 1.73 \mathrm{~m}^{2}$, were routinely referred to primary care clinics for repeated testing $\geq 3$ months later for confirmation of results.

\section{Assessment of geriatric syndromes}

Participants were interviewed further about whether they have geriatric syndromes including depression, dementia, reduced quality of life, fall, and visual disturbance. Depression was assessed based on the major criteria for the diagnosis of major depressive disorder in Diagnostic and Statistical Manual of Mental Disorders $5^{\text {th }}$ version (DSM-V); that is, whether the patient exhibited subjective depressed mood or had anhedonia for most of the day or nearly every day within the last 2 weeks, with 1 point assigned to each criterion (maximal, 2; higher score indicating greater severity) [11]. Quality of life was evaluated using the 5 -item Brief Symptom Rating Scale (BSRS) (score range, 0 to 5 ) as a surrogate, which has been validated in the Taiwanese general population for screening symptom severity and predicting quality of life [12, 13]. The prevalence of fall was assessed by asking whether the patient recalled any experience of fall during the preceding 6 months, while visual disturbance was determined by subjective report. Finally, we assessed cognitive impairment severity through the screening tool AD-8 (score range, 0 to 8), a well-validated tool for cognitive impairment screening with higher scores suggesting more dimensions impairment, the results of which exhibit a high correlation with the Clinical Dementia Rating (CDR) results [14]. All assessment results were ascertained with repetitive queries if participants could not initially clarify their intent.

Analyzing the relationship between self-reported or eGFR-based CKD, CKD complications, and geriatric syndromes

Participant eGFR was calculated based on the CKD Epidemiology Collaboration (CKD-EPI) formula [15] during the initial health examination and subsequent confirmatory testing in the primary care clinics 3 months later. All serum creatinine were measured by the modified Jaffe method, and were IDMS aligned. We defined eGFR-based CKD as those with an eGFR lower than $60 \mathrm{~mL} / \mathrm{min} / 1.73 \mathrm{~m}^{2}$ during both screening 


\section{Kidney Blood Pressure Research}

and repeated testing $\geq 3$ months later, since the existing literature still favors a universal eGFR threshold for diagnosing CKD across age [16]. Instances of self-reported CKD were recognized based on medical history from interview (described above).

We first compared the demographic features, comorbidities, family history of chronic illnesses, physical examination parameters, and laboratory profiles between elderly participants with and without self-reported or eGFR-based CKD. The prevalence and severity of each geriatric syndrome were also compared between groups. This was further supplemented by sub-analyses regarding the influences on CKD complications introduced individually or combining the two CKD assessment methods. Finally, we used multivariate linear regression analyses with backward variable selection to evaluate the relationship between self-reported or eGFR-based CKD and multiple CKD complications, including alterations in serum albumin levels (malnutrition/inflammation), hemoglobin (anemia), and total cholesterol (dyslipidemia), as well as the prevalence or severity of geriatric syndromes.

\section{Statistical Analysis}

All statistical analyses were done using SPSS 18.0 (Chicago, IL, USA). Continuous and categorical variables were compared using an independent $t$-test and a Chi-square test, respectively. Comparisons of more than 2 groups were done using analysis of variance (ANOVA). Two-tailed $p$ values lower than 0.05 were considered statistically significant.

\section{Ethical approval}

The current study has been approved by the institutional review board (No. 201802088RINC), and its protocol adheres to the Declaration of Helsinki. Their identification information was scrambled before data retrieval, and thus informed consent was waived due to data anonymity.

\section{Results}

Over 4 years, a total of 2932 community-dwelling elderly were consecutively enrolled in this study, with a mean age of $74 \pm 7$ years and $45 \%$ male. The most common self-reported comorbidity was hypertension $(49 \%)$, followed by cardiac diseases $(20 \%)$, hyperlipidemia $(18 \%)$, DM $(13 \%)$, and gout $(5 \%)$, with a similar trend for the family history of chronic illnesses. Physical parameters were within the normal ranges (systolic and diastolic BP 128.1 \pm 16.6 and $68.4 \pm 11.1 \mathrm{mmHg}$, PR $70.4 \pm 10.9$, waist circumference $83.4 \pm 9.2 \mathrm{~cm}$ ), as were their laboratory profiles (serum albumin, $4.3 \pm 0.3 \mathrm{mg} / \mathrm{dL}$; hemoglobin $13.5 \pm 1.4 \mathrm{~g} / \mathrm{dL}$ ).

During medical interview, 93 (3.2\%) reported that they had CKD by history. Their mean serum creatinine and eGFR were $0.9 \pm 0.4 \mathrm{mg} / \mathrm{dL}$ and $85 \pm 22 \mathrm{~mL} / \mathrm{min} / 1.73 \mathrm{~m}^{2}$, respectively, and those with eGFR $<60 \mathrm{~mL} / \mathrm{min} / 1.73 \mathrm{~m}^{2}$ were subsequently confirmed in primary care clinic later, resulting in an eGFR-based CKD prevalence of $306(10 \%)$ among these participants. Those with self-reported CKD had significantly higher age $(p<0.01)$, higher prevalence of male $(p<0.01)$, self-reported hypertension $(p<0.01)$, DM $(p=0.02)$, gout $(p<0.01)$, cardiac disease $(p<0.01)$, and higher serum creatinine $(p<0.01)$ while lower serum hemoglobin $(p<0.01)$, albumin $(p<0.01)$, and total cholesterol $(p<0.01)$ than those without (Table 1$)$, all of which were also observed between those with and without eGFR-based CKD. However, those with eGFR-based CKD further had higher BMI $(p<0.01)$, self-reported hyperlipidemia $(p=0.04)$, greater waist circumference $(p<0.01)$ and systolic blood pressure $(p=0.01)$, higher leukocyte count $(p<0.01)$, serum globulin $(p<0.01)$, triglyceride $(p=0.01)$, and fasting glucose $(p<0.01)$ than those without (Table 1), not seen between those with and without self-reported CKD. Significant differences were also observed with regard to the prevalence and severity of geriatric syndromes including depression, cognitive impairment (AD-8 scores), quality of life (BSRS scores), and visual disturbance between those with and without self-reported or eGFR-based CKD, except the prevalence of fall (only between those with and without eGFR-based CKD) (Table 2). 


\section{Kidney Blood Pressure Research}

Table 1. Comparison of clinical features between the elderly with and without self-reported or eGFR-based CKD. BMI, body mass index; BP, blood pressure; CKD, chronic kidney disease; eGFR, estimated glomerular filtration rate; HDL, high density lipoprotein; LDL, low density lipoprotein

\begin{tabular}{|c|c|c|c|c|c|c|}
\hline Clinical features & $\begin{array}{l}\text { With self-reported CKD } \\
\qquad(\mathrm{n}=93)\end{array}$ & $\begin{array}{l}\text { Without self-reported CKD } \\
\qquad(\mathrm{n}=2839)\end{array}$ & $P$ value & $\begin{array}{l}\text { With eGFR-based CKD } \\
(\mathrm{n}=306)\end{array}$ & $\begin{array}{l}\text { Without eGFR-based CKD } \\
\qquad(\mathrm{n}=2626)\end{array}$ & $P$ value \\
\hline \multicolumn{7}{|l|}{ Demographic profile } \\
\hline Age (years) & $76.2 \pm 7.1$ & $73.4 \pm 6.7$ & $<0.01$ & $78.4 \pm 7.2$ & $72.9 \pm 6.4$ & $<0.01$ \\
\hline Gender (male\%) & $58(62)$ & $1250(44)$ & $<0.01$ & $159(52)$ & $1149(44)$ & $<0.01$ \\
\hline BMI $\left(\mathrm{kg} / \mathrm{m}^{2}\right)$ & $23.9 \pm 3.6$ & $23.9 \pm 3.4$ & 0.86 & $24.6 \pm 3.6$ & $23.9 \pm 3.4$ & $<0.01$ \\
\hline Smoking (\%) & $4(4)$ & $141(5)$ & 0.86 & $16(5)$ & $129(5)$ & 0.64 \\
\hline Alcohol consumption (\%) & $22(24)$ & $690(24)$ & 0.88 & $60(20)$ & $652(25)$ & 0.05 \\
\hline Betel nut consumption (\%) & $0(0)$ & $16(1)$ & 0.47 & $3(1)$ & $13(0.5)$ & 0.79 \\
\hline \multicolumn{7}{|l|}{ Other comorbidities by history } \\
\hline Hypertension (\%) & $63(68)$ & $1369(48)$ & $<0.01$ & $214(70)$ & $1218(46)$ & $<0.01$ \\
\hline Diabetes mellitus (\%) & $20(22)$ & $374(13)$ & 0.02 & $77(25)$ & $317(12)$ & $<0.01$ \\
\hline Hyperlipidemia (\%) & $18(19)$ & $509(18)$ & 0.73 & $68(22)$ & $459(17)$ & 0.04 \\
\hline Gout (\%) & $16(17)$ & $134(5)$ & $<0.01$ & $37(12)$ & $113(4)$ & $<0.01$ \\
\hline Cardiac diseases (\%) & $39(42)$ & $546(19)$ & $<0.01$ & $93(30)$ & $492(19)$ & $<0.01$ \\
\hline \multicolumn{7}{|c|}{ Family history of medical illnesses } \\
\hline Hypertension (\%) & $36(39)$ & $978(34)$ & 0.4 & $102(33)$ & $912(35)$ & 0.63 \\
\hline Diabetes mellitus (\%) & $16(17)$ & $533(19)$ & 0.7 & $49(16)$ & $500(19)$ & 0.2 \\
\hline Hyperlipidemia (\%) & $2(2)$ & $28(1)$ & 0.27 & $5(2)$ & $25(1)$ & 0.26 \\
\hline Gout (\%) & $0(0)$ & $8(0)$ & 0.61 & $2(1)$ & $6(0.2)$ & 0.18 \\
\hline Cardiac disease (\%) & $24(26)$ & $352(12)$ & $<0.01$ & $44(14)$ & $332(13)$ & 0.39 \\
\hline CKD (\%) & $1(1)$ & $12(0)$ & 0.35 & $2(1)$ & $11(0.4)$ & 0.56 \\
\hline \multicolumn{7}{|l|}{ Physical parameters } \\
\hline Systolic BP (mmHg) & $125.9 \pm 17.2$ & $128.2 \pm 16.6$ & 0.18 & $130.5 \pm 16.5$ & $127.8 \pm 16.6$ & 0.01 \\
\hline Diastolic BP (mmHg) & $64.9 \pm 11.5$ & $68.6 \pm 11.1$ & $<0.01$ & $66.8 \pm 11.3$ & $68.6 \pm 11.1$ & 0.01 \\
\hline Heart rate (/minute) & $71.5 \pm 12.8$ & $70.3 \pm 10.8$ & 0.32 & $70.8 \pm 11.6$ & $70.3 \pm 10.8$ & 0.46 \\
\hline Waist circumference $(\mathrm{cm})$ & $84.3 \pm 9.1$ & $83.4 \pm 9.2$ & 0.32 & $86.3 \pm 9.5$ & $83.0 \pm 9.1$ & $<0.01$ \\
\hline \multicolumn{7}{|l|}{ Laboratory data } \\
\hline Leuokocyte $\left(x 10^{3} / \mu \mathrm{L}\right)$ & $5.8 \pm 1.5$ & $5.6 \pm 1.5$ & 0.15 & $6.1 \pm 1.6$ & $5.6 \pm 1.5$ & $<0.01$ \\
\hline Platelet $\left(x 10^{3} / \mu \mathrm{L}\right)$ & $191.9 \pm 49.7$ & $210.9 \pm 54$ & $<0.01$ & $204 \pm 54.1$ & $211 \pm 53.9$ & 0.03 \\
\hline Hemoglobin (g/dL) & $12.8 \pm 1.9$ & $13.5 \pm 1.3$ & $<0.01$ & $12.7 \pm 1.7$ & $13.6 \pm 1.3$ & $<0.01$ \\
\hline Albumin (mg/dL) & $4.2 \pm 0.3$ & $4.3 \pm 0.3$ & $<0.01$ & $4.2 \pm 0.3$ & $4.3 \pm 0.3$ & $<0.01$ \\
\hline Globulin (mg/dL) & $2.8 \pm 0.4$ & $2.8 \pm 0.4$ & 0.94 & $2.9 \pm 0.4$ & $2.8 \pm 0.4$ & $<0.01$ \\
\hline Urea nitrogen (mg/dL) & $27.1 \pm 14.6$ & $16.8 \pm 5$ & $<0.01$ & $25.5 \pm 10.5$ & $16.1 \pm 4$ & $<0.01$ \\
\hline Creatinine $(\mathrm{mg} / \mathrm{dL})$ & $1.7 \pm 1.7$ & $0.9 \pm 0.3$ & $<0.01$ & $1.5 \pm 1$ & $0.8 \pm 0.2$ & $<0.01$ \\
\hline $\mathrm{eGFR}\left(\mathrm{ml} / \mathrm{min} / 1.73 \mathrm{~m}^{2}\right)$ & $58.1 \pm 28.2$ & $85.7 \pm 21$ & $<0.01$ & $47.2 \pm 11.8$ & $89.4 \pm 18$ & $<0.01$ \\
\hline Fasting glucose (mg/dL) & $101.1 \pm 20.8$ & $100.6 \pm 20.6$ & 0.8 & $104.9 \pm 28.8$ & $100.1 \pm 19.3$ & $<0.01$ \\
\hline Total cholesterol $(\mathrm{g} / \mathrm{dL})$ & $173.7 \pm 26$ & $184 \pm 33.4$ & $<0.01$ & $175.8 \pm 31.4$ & $184.3 \pm 33.3$ & $<0.01$ \\
\hline LDL (mg/dL) & $101.9 \pm 23.3$ & $110.9 \pm 27.9$ & $<0.01$ & $104.6 \pm 26.7$ & $111.3 \pm 27.9$ & $<0.01$ \\
\hline HDL (mg/dL) & $50.4 \pm 11.8$ & $51.9 \pm 13.1$ & 0.27 & $48 \pm 13.4$ & $52.3 \pm 12.9$ & $<0.01$ \\
\hline Triglyceride (mg/dL) & $116.7 \pm 73.9$ & $119.7 \pm 64.4$ & 0.66 & $129.1 \pm 62.2$ & $118.5 \pm 64.9$ & 0.01 \\
\hline Uric acid (mg/dL) & $6.4 \pm 1.6$ & $5.8 \pm 1.4$ & $<0.01$ & $6.9 \pm 1.6$ & $5.7 \pm 1.3$ & $<0.01$ \\
\hline
\end{tabular}

Table 2. Comparison of geriatric syndromes between the elderly with and without self-reported or eGFRbased CKD. BSRS, Brief Symptom Rating Scale; CKD, chronic kidney disease; eGFR, estimated glomerular filtration rate

\begin{tabular}{|c|c|c|c|c|c|c|}
\hline Geriatric syndromes & $\begin{array}{l}\text { With self-reported } \\
\text { CKD }(n=93)\end{array}$ & $\begin{array}{l}\text { Without self-reported } \\
\text { CKD }(\mathrm{n}=2839)\end{array}$ & $P$ value & $\begin{array}{l}\text { With creatinine-based } \\
\text { CKD }(n=306)\end{array}$ & $\begin{array}{l}\text { Without creatinine-based } \\
\text { CKD }(\mathrm{n}=2626)\end{array}$ & $P$ value \\
\hline Depression scores & $0.4 \pm 0.7$ & $0.2 \pm 0.5$ & $<0.01$ & $0.3 \pm 0.6$ & $0.2 \pm 0.5$ & $<0.01$ \\
\hline AD-8 scores & $1 \pm 2.1$ & $0.6 \pm 1.3$ & $<0.01$ & $0.8 \pm 1.6$ & $0.5 \pm 1.3$ & $<0.01$ \\
\hline BSRS scores & $3.5 \pm 4$ & $2 \pm 2.8$ & $<0.01$ & $2.8 \pm 3.4$ & $1.9 \pm 2.6$ & $<0.01$ \\
\hline Fall & $7(8)$ & $141(5)$ & 0.27 & $46(8)$ & $102(4)$ & $<0.01$ \\
\hline Visual disturbance & $18(19)$ & 297 (10) & $<0.01$ & 99 (17) & $216(9)$ & $<0.01$ \\
\hline
\end{tabular}

We subsequently analyzed clinical parameters and the prevalence or severity of geriatric syndromes, after dividing participants into "dual negative","eGFR positive", "selfreport positive", and "dual positive" groups (Table 3). We found that significant betweengroup differences existed regarding demographic profiles (age, gender, BMI), self-reported comorbidities (except hyperlipidemia), physical parameters (BP and waist circumferences), laboratory data, and geriatric syndromes (except the prevalence of fall). A diagram summarizing the clinical features and geriatric syndromes that had the highest values, prevalence, or the greatest severity among the 4 groups is provided in Fig. 1 . Elderly patients with dual positive CKD had the highest prevalence of several morbidities, the worst renal function, and the highest degree of laboratory-based inflammation (leukocyte and serum globulin levels) compared to others, while those with only self-reported CKD had a higher prevalence of comorbid gout and cardiac diseases.

We further investigated the individual and combinatorial influences introduced by self-reported and eGFR-based CKD on complications related to CKD. The presence of selfreported and eGFR-based CKD each negatively affected serum albumin, hemoglobin, and total cholesterol levels $(p<0.01$ for comparisons of each parameter between the 4 groups using 


\section{Kidney Blood Pressure Research}

Table 3. Comparison of clinical features and geriatric syndromes between the elderly with and without eGFR-based CKD (E) or self-reported CKD (S). BMI, body mass index; BP, blood pressure; CKD, chronic kidney disease; eGFR, estimated glomerular filtration rate; HDL, high density lipoprotein; LDL, low density lipoprotein

\begin{tabular}{|c|c|c|c|c|c|}
\hline Clinical features & $E+/ S+(n=48)$ & $E-/ S+(n=45)$ & $E+/ S-(n=258)$ & $E-/ S-(n=2581)$ & $P$ value \\
\hline \multicolumn{6}{|l|}{ Demographic profile } \\
\hline Age (years) & $78.3 \pm 7$ & $74 \pm 6.6$ & $78.4 \pm 7.3$ & $72.9 \pm 6.4$ & $<0.01$ \\
\hline Gender (male\%) & $33(69)$ & $25(56)$ & $126(49)$ & $1124(44)$ & $<0.01$ \\
\hline BMI $\left(\mathrm{kg} / \mathrm{m}^{2}\right)$ & $23.6 \pm 3.3$ & $24.2 \pm 3.8$ & $24.8 \pm 3.6$ & $23.9 \pm 3.4$ & $<0.01$ \\
\hline Smoking (\%) & $1(2)$ & $3(7)$ & $15(6)$ & $118(5)$ & 0.59 \\
\hline Alcohol consumption (\%) & $11(23)$ & $11(24)$ & 49 (19) & $641(25)$ & 0.21 \\
\hline Betel nut consumption (\%) & $0(0)$ & $0(0)$ & $2(1)$ & $14(1)$ & 0.86 \\
\hline \multicolumn{6}{|l|}{ Other comorbidities by history } \\
\hline Hypertension (\%) & $36(75)$ & $27(60)$ & $178(69)$ & $1191(46)$ & $<0.01$ \\
\hline Diabetes mellitus (\%) & $15(31)$ & $5(11)$ & $62(24)$ & $312(12)$ & $<0.01$ \\
\hline Hyperlipidemia (\%) & $8(17)$ & $10(22)$ & $60(23)$ & 449 (17) & 0.11 \\
\hline Gout (\%) & $8(17)$ & $8(18)$ & $29(11)$ & $105(4)$ & $<0.01$ \\
\hline Cardiac disease (\%) & $21(44)$ & $18(40)$ & $72(28)$ & $474(18)$ & $<0.01$ \\
\hline \multicolumn{6}{|c|}{ Family history of medical illnesses } \\
\hline Hypertension (\%) & $15(31)$ & $21(47)$ & $87(34)$ & $891(35)$ & 0.36 \\
\hline Diabetes mellitus (\%) & $10(21)$ & $6(13)$ & $39(15)$ & $494(19)$ & 0.32 \\
\hline Hyperlipidemia (\%) & $1(2)$ & $1(2)$ & $4(2)$ & $24(1)$ & 0.55 \\
\hline Gout (\%) & $0(0)$ & $0(0)$ & $2(1)$ & $6(0)$ & 0.42 \\
\hline Cardiac disease $(\%)$ & $11(23)$ & $13(29)$ & $33(13)$ & $319(12)$ & $<0.01$ \\
\hline CKD $(\%)$ & $1(2)$ & $0(0)$ & $1(0)$ & $11(0)$ & 0.37 \\
\hline \multicolumn{6}{|l|}{ Physical parameters } \\
\hline Systolic BP (mmHg) & $126.6 \pm 18.3$ & $125 \pm 16.1$ & $131.3 \pm 16$ & $127.9 \pm 16.6$ & $<0.01$ \\
\hline Diastolic BP (mmHg) & $64.3 \pm 13$ & $65.4 \pm 9.8$ & $67.3 \pm 10.9$ & $68.7 \pm 11.1$ & $<0.01$ \\
\hline Heart rate (/minute) & $71.6 \pm 13.4$ & $71.4 \pm 12.2$ & $70.7 \pm 11.2$ & $70.3 \pm 10.8$ & 0.74 \\
\hline Waist circumference $(\mathrm{cm})$ & $84.1 \pm 9$ & $84.5 \pm 9.3$ & $86.7 \pm 9.5$ & $83 \pm 9.1$ & $<0.01$ \\
\hline \multicolumn{6}{|l|}{ Laboratory data } \\
\hline Leuokocyte $\left(\mathrm{x} 10^{6} / \mu \mathrm{L}\right)$ & $6.1 \pm 1.7$ & $5.5 \pm 1.2$ & $6 \pm 1.6$ & $5.6 \pm 1.5$ & $<0.01$ \\
\hline Platelet $\left(\mathrm{x} 10^{3} / \mu \mathrm{L}\right)$ & $190.3 \pm 50.1$ & $193.6 \pm 49.7$ & $206.5 \pm 54.5$ & $211.3 \pm 54$ & $<0.01$ \\
\hline Hemoglobin $(\mathrm{g} / \mathrm{dL})$ & $12.1 \pm 1.9$ & $13.5 \pm 1.6$ & $12.8 \pm 1.6$ & $13.6 \pm 1.3$ & $<0.01$ \\
\hline Albumin (mg/dL) & $4.1 \pm 0.3$ & $4.3 \pm 0.3$ & $4.2 \pm 0.3$ & $4.3 \pm 0.3$ & $<0.01$ \\
\hline Globulin (mg/dL) & $2.9 \pm 0.5$ & $2.7 \pm 0.3$ & $2.9 \pm 0.4$ & $2.8 \pm 0.4$ & $<0.01$ \\
\hline Urea nitrogen $(\mathrm{mg} / \mathrm{dL})$ & $35.1 \pm 15.5$ & $18.6 \pm 6.9$ & $23.8 \pm 8.1$ & $16.1 \pm 3.9$ & $<0.01$ \\
\hline Creatinine $(\mathrm{mg} / \mathrm{dL})$ & $2.5 \pm 2.1$ & $0.9 \pm 0.2$ & $1.4 \pm 0.5$ & $0.8 \pm 0.2$ & $<0.01$ \\
\hline eGFR (ml/min $\left./ 1.73 \mathrm{~m}^{2}\right)$ & $36.8 \pm 16.6$ & $83 \pm 15.5$ & $49.2 \pm 9.5$ & $89.5 \pm 18$ & $<0.01$ \\
\hline Fasting glucose $(\mathrm{mg} / \mathrm{dL})$ & $106.8 \pm 25.7$ & $94.6 \pm 10.2$ & $104.5 \pm 29.4$ & $100.2 \pm 19.4$ & $<0.01$ \\
\hline Total cholesterol $(\mathrm{g} / \mathrm{dL})$ & $171.3 \pm 25.2$ & $176.4 \pm 26.9$ & $176.6 \pm 32.4$ & $184.4 \pm 33.4$ & $<0.01$ \\
\hline $\mathrm{LDL}(\mathrm{mg} / \mathrm{dL})$ & $99 \pm 22.4$ & $105.2 \pm 24$ & $105.7 \pm 27.4$ & $111.4 \pm 27.9$ & $<0.01$ \\
\hline HDL (mg/dL) & $48 \pm 11.8$ & $53.1 \pm 11.5$ & $48 \pm 13.7$ & $52.3 \pm 13$ & $<0.01$ \\
\hline Triglyceride (mg/dL) & $127.8 \pm 87$ & $104 \pm 53.6$ & $129.4 \pm 56.6$ & $118.7 \pm 65.1$ & 0.02 \\
\hline Uric acid (mg/dL) & $6.8 \pm 1.8$ & $6 \pm 1.3$ & $6.9 \pm 1.5$ & $5.7 \pm 1.3$ & $<0.01$ \\
\hline \multicolumn{6}{|l|}{ Geriatric syndromes } \\
\hline Depression scores & $0.5 \pm 0.8$ & $0.3 \pm 0.6$ & $0.2 \pm 0.5$ & $0.2 \pm 0.5$ & $<0.01$ \\
\hline AD-8 scores & $1.2 \pm 2.3$ & $0.9 \pm 1.8$ & $1 \pm 1.7$ & $0.5 \pm 1.3$ & $<0.01$ \\
\hline BSRS scores & $3.6 \pm 3.8$ & $3.4 \pm 4.3$ & $2.3 \pm 2.9$ & $2 \pm 2.7$ & $<0.01$ \\
\hline Fall & $4(8)$ & $3(7)$ & $18(7)$ & $123(5)$ & 0.41 \\
\hline Visual disturbance & $9(19)$ & $9(20)$ & $57(22)$ & 240 (9) & $<0.01$ \\
\hline
\end{tabular}

ANOVA) (Fig. 2A to 2D), suggesting that both methods of determining CKD play a different but synergistic role in influencing patient outcomes. Similar findings were observed for the severity of geriatric syndromes including cognitive impairment (AD-8 scores) and quality of life (BSRS scores) $(p<0.01)$ (Fig. 2E and 2F). 


\section{Kidney Blood Pressure Research}

Fig. 1. A summary diagram illustrating variables exhibiting the highest values, highest prevalence, or the greatest severity (if geriatric syndromes) among different groups of older participants, with and without self-report or eGFR-based CKD.* Quality of life, cognitive impairment, depression \& Visual disturbance. Alb, albumin; BMI, body mass index; BUN, blood urea nitrogen; CKD, chronic kidney disease; Cre, creatinine; DM, diabetes mellitus; eGFR, estimated glomerular filtration rate; $\mathrm{Hb}$, hemoglobin; HTN, hypertension; LDL, low density lipoprotein cholesterol; T-cho, total cholesterol; WBC, white blood cell.

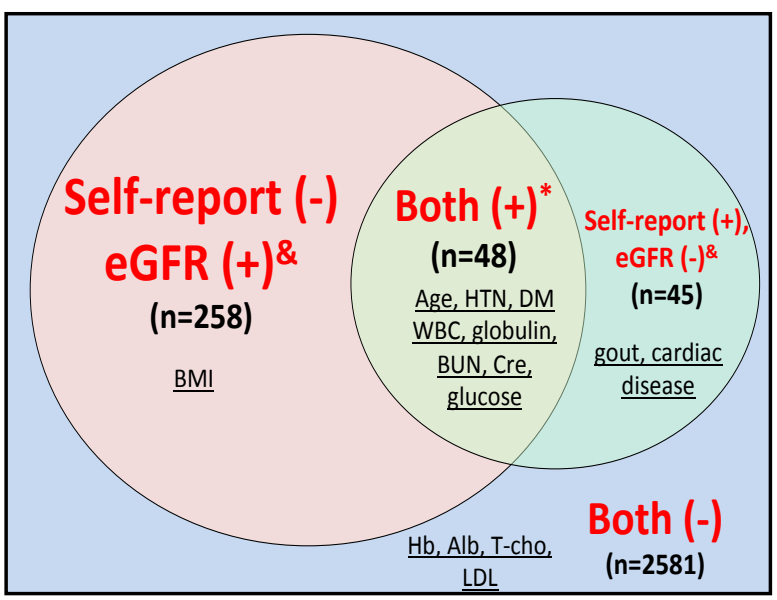

Fig. 2. CKD-related complications based on laboratory profiles (A, serum albumin; B, hemoglobin; C, total cholesterol; D, leukocyte count) and geriatric syndrome assessment (E, cognitive scores based on AD-8 results; $\mathrm{F}$, quality of life based on BSRS scores) among different groups of elderly participants. BSRS, brief symptom rating scale; CKD, chronic kidney disease; eGFR, estimated glomerular filtration rate.

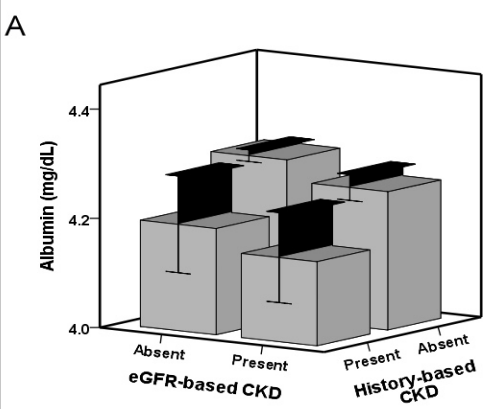

C

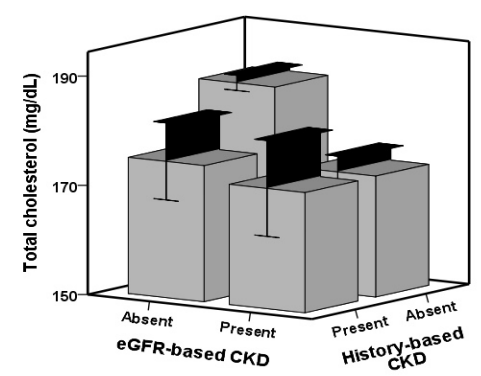

$E$

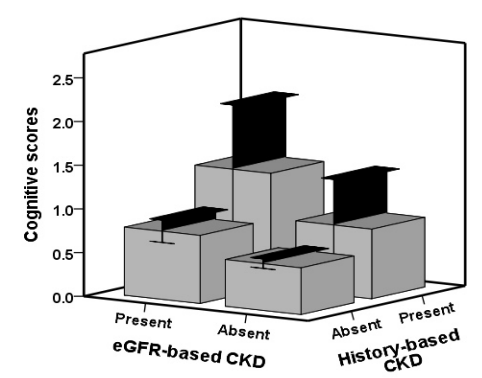

B

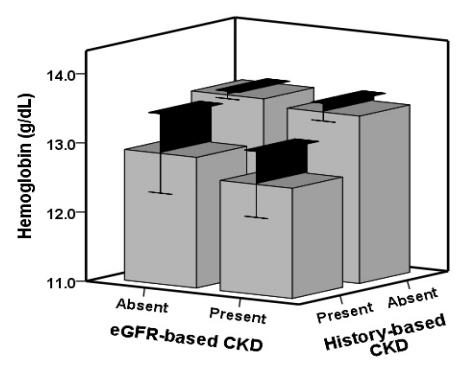

D

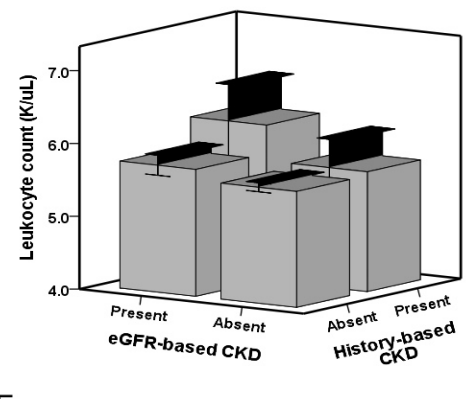

$\mathrm{F}$

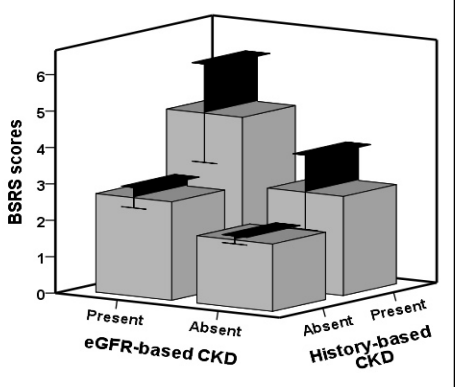




\section{Kidney Blood Pressure Research}

Finally, we performed multivariate linear regression analyses to examine the association between the two CKD assessment methods, CKD complications, and geriatric syndromes. Adjusting for demographic profile, other comorbidities, and physical parameters, selfreported CKD stood out independently as a negative determinant for serum albumin $(\beta=$ $-0.04, \mathrm{t}=-2.46, p=0.01)$ and hemoglobin $(\beta=-0.08, \mathrm{t}=-5.44, p<0.01)$ but not eGFR-based CKD, while the latter was a negative determinant for total cholesterol $(\beta=-0.12, t=-6.79, p<$ 0.01 ) but not the former. Both assessment methods were independent predictors for higher BSRS scores, a surrogate for poor quality of life (for self-reported CKD, $\beta=0.08, \mathrm{t}=4.58, p$ $<0.01$; for eGFR-based, $\beta=0.12, \mathrm{t}=6.19, p<0.01$ ) and higher depression scores (for selfreported CKD, $\beta=0.06, \mathrm{t}=3.26, p<0.01$; for eGFR-based, $\beta=0.06, \mathrm{t}=2.98, p<0.01$ ), while only eGFR-based CKD was significant predictor for higher AD-8 scores, or more cognitive impairment $(\beta=0.04, \mathrm{t}=2.26, p=0.02)$.

\section{Discussion}

In this study, we found that older adults with self-reported CKD exhibited a different risk factor combination compared to those with eGFR-based CKD, and the prevalence or severity of geriatric syndromes was higher among those with either form of CKD. Elderly with dual positive CKD (i.e. recognized by both self-report and reduced eGFR) were more likely to have multimorbidity, low eGFR and higher inflammation. After accounting for demographic profile, comorbidities, and physical parameters, self-reported and eGFR-based CKD each had independent association with different types of CKD-related complications and geriatric syndromes.

We observed that the prevalence of self-report CKD (3.2\%) was low among the elderly, a finding that would not be unexpected based on the following reasons. First, studies from large population-based screening of CKD already showed that the prevalence of self-report CKD could be very low despite a high prevalence of true CKD. Findings from the KEEP cohort suggested that the prevalence of CKD among 100,000 participants was $28.7 \%$ but only $6.7 \%$ had self-report CKD [17]. In the Reasons for Geographical and Racial Difference in Stroke (REGARDS) cohort, only 6.7\% of those with CKD reported that they had kidney disease [18]. The low prevalence of self-report CKD in these studies, ranging from $2 \%$ to $7 \%$, lends support to the credibility of our findings. Second, our participants were recruited from communitydwelling functionally intact elderly receiving health examination during the study period, and these older participants had relatively little morbidity (see Table $1,<15 \%$ with DM or heart disease). These factors may underlie the low prevalence of self-report CKD among this study.

Discrepancy between self-reported history and health records is most commonly reported in the documentation of prescribed medications [19-21], but under-recognized in the reporting of morbidity. Prior studies revealed that higher age and more severe comorbidities were significant risk factors for inaccurate history reporting [22, 23]; based on these data, it is likely that among older adults, self-reported CKD is not equivalent to true CKD. Plausible reasons to explain the different detection rates between self-reported and eGFR-based CKD include the limited health literacy for the elderly with CKD, and the erroneous belief that they have concurrent CKD brought by their pre-existing comorbidities. A systematic review identified that $20 \%$ to $25 \%$ CKD patients had poor health literacy, particularly those with more severe comorbidity and low serum albumin [24], while others suggested that lower health literacy significantly correlated with poor CKD awareness [25]. These factors may contribute to a "false positive" or false negative result for CKD during medical interview.

In this study, we found that patients with self-reported but without eGFR-based CKD had several interesting features compared to those of the other groups (Fig. 1), including a higher prevalence of gout and cardiac diseases, suggestive of some metabolic disturbance 


\section{Kidney Blood Pressure Research}

among these participants. Evidence suggests that aging with or without CKD is accompanied by increased body adiposity, decreased lean mass, and a higher prevalence of metabolic syndrome [26-28]. Serum uric acid is reportedly associated with a concentration-dependent increased risk of incident CKD or worsening renal function in several studies $[29,30]$, while higher age further aggravates the scenario. We surmise that self-reported CKD in the absence of low eGFR can be a surrogate for pre-existing metabolic disturbance predisposing the individual for subsequent CKD development. Having self-reported CKD may represent a transitory status before the onset of laboratory-based CKD, permitting targeted intervene to reduce incident CKD in the future. There are now studies suggesting that treatment for gout and hyperuricemia can successfully lower the subsequent risk of CKD development and these diseases per se influence the risk of acute kidney injury [31-34].

Though our findings, based on a large group of older adults, may have their clinical implications, several limitations should be considered when we extrapolate these results to other settings. The associations we observed between CKD assessed by different approaches and various endpoints may not be causative in origin, as we do not have longitudinal followup results at this time. A cohort study would be needed from this perspective. According to past studies, the accuracy of self-report comorbidities can vary depending on the types of diseases, and the comorbidity profile in this study was also based on self-report. However, most studies revealed that the accuracy of self-report cardiometabolic diseases was high, while that of symptom-based disease was low [6, 7]. In this study, we assessed comorbidities related to cardiometabolic diseases only (Table 1). In order to verify their accuracy, we further ascertained this issue based on the rate of concordance between self-report hypertension, $\mathrm{DM}$ and the prescription of anti-hypertensive and anti-diabetic medications. We found that 92.4\% and $90.6 \%$ self-report hypertensive and diabetic patients used at least one antihypertensive and anti-diabetic medication, respectively. These findings support the accuracy of other self-report comorbidities assessed in this study. In addition, cognitive impairment should also be considered before interpreting our findings of self-reported comorbidities, but we believe that its influence is minimal, since our elderly participants had relatively low severity of cognitive impairment, manifesting as low AD-8 scores on average $(0.6 \pm 1.4$ out of 8). Finally, although there are significant differences in multiple biochemical parameters between groups with different CKD assessments, results in each group are still within the normal range of the tested variables. Thus, our results are most likely applicable to healthy elderly who live in communities. Nonetheless, the influence of this discrepancy on patient outcomes has not been explored before. Our findings of the differential impact on geriatric syndrome severity may pave the way toward better understanding of the clinical importance of how CKD is recognized in the elderly.

\section{Conclusion}

In summary, using data from a large cohort of community-dwelling older adults, we found that self-reported and eGFR-based CKD might have different clinical meanings, their associations manifesting with a different subset of comorbidities and CKD-related complications. Self-reported and eGFR-based CKD may act synergistically regarding their influences on CKD-related complications, the prevalence and severity of multiple geriatric syndromes. Since self-reported CKD may serve as a surrogate for pre-existing risk factor for true CKD, an earlier emphasis of risk mitigation strategy and regular medical follow-up for older adults with self-reported but without eGFR-based CKD is recommended in order to prevent the emergence of CKD in the future. 


\section{Kidney \\ Blood Pressure \\ Research}

Chao et al.: Self-Report and eGFR-Based CKD

\section{Abbreviations}

ANOVA, analysis of variance; BMI, body mass index; BP, blood pressure; BSRS, Brief Symptom Rating Scale; CDR, Clinical Dementia Rating; CKD, chronic kidney disease; CKD-EPI, CKD Epidemiology Collaboration; DM, diabetes mellitus; DSM-V, Diagnostic and Statistical Manual of Mental Disorders $5^{\text {th }}$ version; eGFR, estimated glomerular filtration rate; ESRD, end-stage renal disease; PR, pulse rate; UN, urea nitrogen

\section{Acknowledgements}

We thank the assistants of the T1 project in the National Taiwan University Hospital BeiHu branch for their help in data collection and trimming. An abstract of this study has been accepted in Kidney Week 2018. Otherwise, this manuscript has not been published previously in whole or in part. The study is financially sponsored by National Taiwan University Hospital BeiHu branch, and Ministry of Science and Technology, Taiwan (MOST 106-2314-B-002-132-MY2). The sponsors have no role in the study design, data collection, analysis, and result interpretation of this study. Study design: CTC, SIC, JWH; Data analysis: CTC, SIC, DSH, JWH; Article drafting: CTC, KCY, JKP, CML, SIC, DSH, JWH; All authors approved the final version of the manuscript.

\section{Disclosure Statement}

The authors have no relevant financial or non-financial competing interests to declare in relation to this manuscript.

\section{References}

1 Jha V, Garcia-Garcia G, Iseki K, Li Z, Naicker S, Plattner B, Saran R, Wang AY, Yang CW: Chronic kidney disease: global dimension and perspectives. Lancet 2013;382:260-272.

2 Lozano R, Naghavi M, Foreman K, Lim S, Shibuya K, Aboyans V, Abraham J, Adair T, Aggarwal R, Ahn SY, et al: Global and regional mortality from 235 causes of death for 20 age groups in 1990 and 2010: a systematic analysis for the Global Burden of Disease Study 2010. Lancet 2012;380:2095-2128.

-3 Plantinga LC, Boulware L, Coresh J, Stevens LA, Miller ER 3rd, Saran R, Messer KL, Levey AS, Powe NR: Patient awareness of chronic kidney disease: Trends and predictors. Arch Intern Med 2008;168:2268-2275.

4 Vassalotti JA, Li S, McCullough PA, Bakris GL: Kidney Early Evaluation Program: A Community-Based Screening Approach to Address Disparities in Chronic Kidney Disease. Semin Nephrol 2010;30:66-73.

5 Hwang SJ, Tsai JC, Chen HC: Epidemiology, impact and preventive care of chronic kidney disease in Taiwan. Nephrology (Carlton) 2010;15:3-9.

-6 Bush TL, Miller SR, Golden AL, Hale WE: Self-report and medical record report agreement of selected medical conditions in the elderly. Am J Public Health 1989;79:1554-1556.

7 Skinner KM, Miller DR, Lincoln E, Lee A, Kazis LE: Concordance between respondent self-reports and medical records for chronic conditions: experience from the Veterans Health Study. J Ambul Care Manage 2005;28:102-110.

8 Tsai AC, Chang TL: Quality issues of self-report of hypertension: analysis of a population representative sample of older adults in Taiwan. Arch Gerontol Geriatr 2012;55:338-342.

-9 Koroukian SM, Warner DF, Owusu C, Given CW: Multimorbidity redefined: prospective health outcomes and the cumulative effect of co-occurring conditions. Prev Chronic Dis 2015;12E55.

10 Koroukian SM, Schiltz N, Warner DF, Sun J, Bakaki PM, Smyth KA, Stange KC, Given CW: Combinations of chronic conditions, functional limitations, and geriatric syndromes that predict health outcomes. J Gen Intern Med 2016;31:630-637. 


\section{Kidney \\ Blood Pressure Research}

Chao et al:: Self-Report and eGFR-Based CKD

11 McGlinchey JB, Zimmerman M, Young D, Chelminski I: Diagnosing Major Depressive Disorder VIII: Are Some Symptoms Better Than Others? J Nerv Ment Dis 2006;194:785-790.

12 Chen $\mathrm{H}, \mathrm{Wu}$ C, Lee Y, Liao S, Lee M: Validity of the five-item Brief Symptom Rating Scale among subjects admitted for general health screening. J Formos Med Assoc 2005;104:824-829.

13 Lu I-C, Yen Jean M-C, Lei S-M, Cheng H-H, Wang J-D: BSRS-5 (5-item Brief Symptom Rating Scale) scores affect every aspect of quality of life measured by WHOQOL-BREF in healthy workers. Qual Life Res 2011;20:1469-1475.

14 Galvin JE, Roe CM, Powlishta KK, Coats MA, Muich SJ, Grant E, Miller JP, Storandt M, Morris JC: The AD8: A brief informant interview to detect dementia. Neurology 2005;65:559-564.

15 Levey AS, Stevens LA, Schmid CH, Zhang YL, Castro AF 3rd, Feldman HI, Kusek JW, Eggers P, Van Lente F, Greene T, Coresh J; CKD-EPI (Chronic Kidney Disease Epidemiology Collaboration). A new equation to estimate glomerular filtration rate. Ann Intern Med 2009;150:604-612.

16 Hui X, Matsushita K, Sang Y, Ballew SH, Fülöp T, Coresh J: CKD and Cardiovascular Disease in the Atherosclerosis Risk in Communities (ARIC) Study: Interactions With Age, Sex, and Race. Am J Kidney Dis 2013;62:691-702.

17 Vassalotti JA, Li S, Chen SC, Collins AJ: Screening populations at increased risk of CKD: the Kidney Early Evaluation Program (KEEP) and the public health problem. Am J Kidney Dis 2009;53:S107-S114.

$\checkmark 18$ McClellan WM, Newsome BB, McClure LA, Cushman M, Howard G, Audhya P, Abramson JL, Warnock DG: Chronic kidney disease is often unrecognized among patients with coronary heart disease: the REGARDS cohort study. Am J Nephrol 2009;29:10-17.

19 Cornish PL, Knowles SR, Marchesano R, Tam V, Shadowitz S, Juurlink DN, Etchells EE: Unintended medication discrepancies at the time of hospital admission. Arch Intern Med 2005;165:424-429.

20 Lee KP, Nishimura K, Ngu B, Tieu L, Auerbach AD: Predictors of Completeness of Patients' Self-reported Personal Medication Lists and Discrepancies With Clinic Medication Lists. Ann Pharmacother 2014;48:168177.

21 Monte AA, Anderson P, Hoppe JA, Weinshilboum RM, Vasiliou V, Heard KJ: Accuracy of Electronic Medical Record Medication Reconciliation in Emergency Department Patients. J Emerg Med 2015;49:78-84.

22 Mukerji SS, Duffy SA, Fowler KE, Khan M, Ronis DL, Terrell JE: Comorbidities in head and neck cancer: Agreement between self-report and chart review. Otolaryngol Head Neck Surg 2007;136:536-542.

23 Klabunde CN, Reeve BB, Harlan LC, Davis WW, Potosky AL: Do Patients Consistently Report Comorbid Conditions Over Time?: Results From the Prostate Cancer Outcomes Study. Med Care 2005;43:391-400.

-24 Fraser SDS, Roderick PJ, Casey M, Taal MW, Yuen HM, Nutbeam D: Prevalence and associations of limited health literacy in chronic kidney disease: a systematic review. Nephrol Dial Transplant 2013;28:129-137.

25 Wright JA, Wallston KA, Elasy TA, Ikizler TA, Cavanaugh KL: Development and Results of a Kidney Disease Knowledge Survey Given to Patients With CKD. Am J Kidney Dis 2011;57:387-395.

26 Dominguez LJ, Barbagallo M: The biology of the metabolic syndrome and aging. Curr Opin Clin Nutr Metab Care 2016;19:5-11.

27 Chao CT, Hsu YH, Chang PY, He YT, Ueng RS, Lai CF, Chiang CK, Huang JW, Huang SJ: Simple self-report FRAIL scale might be more closely associated with dialysis complications than other frailty screening instruments in rural chronic dialysis patients. Nephrology 2015;20:321-328.

28 Chao CT, Chan DC, Huang JW: Frail phenotype might be associated with higher appendicular but not truncal fat among end-stage renal disease patients. J Pain Symptom Manage 2017;53:e1-e4.

29 Chonchol M, Shlipak MG, Katz R, Sarnak MJ, Newman AB, Siscovick DS, Kestenbaum B, Carney JK, Fried LF: Relationship of Uric Acid With Progression of Kidney Disease. Am J Kidney Dis 2007;50:239-247.

-30 Chien K-L, Lin H-J, Lee B-C, Hsu H-C, Lee Y-T, Chen M-F: A Prediction Model for the Risk of Incident Chronic Kidney Disease. Am J Med 2010;123:836-846.

-31 Friedman AN, Wahed AS, Wang J, Courcoulas AP, Dakin G, Hinojosa MW, Kimmel PL, Mitchell JE, Pomp A, Pories WJ, Purnell JQ le Roux C, Spaniolas K, Steffen KJ, Thirlby R, Wolfe B: Effect of Bariatric Surgery on CKD Risk. J Am Soc Nephrol 2018;29:1289-1300.

-32 Kim Y, Shin S, Kim K, Choi S, Lee K: Effect of Urate Lowering Therapy on Renal Disease Progression in Hyperuricemic Patients with Chronic Kidney Disease. J Rheumatol 2015;42:2143-2148.

33 Chao CT, Wang J, Wu HY, Huang JW, Chien KL: Age modifies the risk factor profiles for acute kidney injury among recently diagnosed type 2 diabetic patients: a population-based study. Geroscience 2018;40:201217.

34 Chao CT, Wang J, Chien KL: Both pre-frailty and frailty increase healthcare utilization and adverse health outcomes in patients with type 2 diabetes mellitus. Cardiovasc Diabetol 2018;17:130. 